\title{
Visual working memory contaminates perception
}

\author{
Min-Suk Kang • Sang Wook Hong • Randolph Blake • \\ Geoffrey F. Woodman
}

Published online: 29 June 2011

(C) Psychonomic Society, Inc. 2011

\begin{abstract}
Indirect evidence suggests that the contents of visual working memory may be maintained within sensory areas early in the visual hierarchy. We tested this possibility using a well-studied motion repulsion phenomenon in which perception of one direction of motion is distorted when another direction of motion is viewed simultaneously. We found that observers misperceived the actual direction of motion of a single motion stimulus if, while viewing that stimulus, they were holding a different motion direction in visual working memory. Control experiments showed that none of a variety of alternative explanations could account for this repulsion effect induced by working memory. Our findings provide compelling evidence that visual working memory representations directly interact with the same neural mechanisms as those involved in processing basic sensory events.
\end{abstract}

Keywords Visual working memory Visual perception

M.-S. Kang · S. W. Hong • R. Blake · G. F. Woodman

Department of Psychology \& Vanderbilt Vision Research Center, Vanderbilt University,

Nashville, USA

R. Blake

Department of Brain and Cognitive Sciences,

Seoul National University,

Seoul, Korea

M.-S. Kang $(\bowtie)$

Department of Psychology, Vanderbilt University,

Wilson Hall PMB 4078172301 Vanderbilt Place,

Nashville, TN 37240-7817, USA

e-mail: m.suk.kang@gmail.com

\section{Introduction}

People are able to hold and manipulate visual information in a limited-capacity working memory system (Luck \& Vogel, 1997; Miller, 1956) that is believed to be related to multiple cognitive abilities, including intelligence (Desimone \& Duncan, 1995; Fukuda, Vogel, Mayr, \& Awh, 2010) and emotional self-regulation (Schmeichel \& Demaree, 2010). Remarkably, recent brain-imaging work suggests that the contents of visual working memory might actually be represented within retinotopically organized visual areas, including the primary visual cortex (Harrison \& Tong, 2009; Serences, Ester, Vogel, \& Awh, 2009). If neural processes responsible for visual perception and visual working memory are indeed so intertwined, we would expect the contents of memory to be reflected in our perceptual experiences. It is known that the contents of working memory can bias the allocation of attentional resources (Desimone \& Duncan, 1995; Soto, Hodsoll, Rotshtein, \& Humphreys, 2008; Woodman \& Luck, 2007), but whether perception itself is altered by those contents remains an unanswered question. Because sensory neurons are known to multiplex (Panzeri, Brunel, Logothetis, \& Kayser, 2010), it is possible that the cognitive processes of perception and the storage of information in working memory do not directly interact even though the same neurons are involved in both processes. In this study, we directly addressed the question of whether working memory representations change the appearance of new sensory inputs, using psychophysical techniques to measure observers' perception of motion while holding a different motion direction in visual working memory.

When two superimposed sets of random dots move in different directions, the perceived angular separation of the two directions of motion are exaggerated relative to their 
actual angular separation. This well-studied perceptual illusion is called motion repulsion (Hiris \& Blake, 1996; Marshak \& Sekuler, 1979). We reasoned that if representations of motion in visual working memory directly influence sensory inputs, an observer's motion perception should systematically deviate away from a direction of the motion represented in visual working memory. The motion repulsion effect is especially well suited for studying interactions between perception and working memory, for two reasons. First, the neural mechanisms mediating visual working memory retention have been systematically investigated with random-dot motion stimuli in humans and nonhuman primates (Blake, Cepeda, \& Hiris, 1997; Pasternak \& Greenlee, 2005). Second, it is difficult to explain the repulsion effect on the basis of demand characteristics, an experimental artifact where participants form an interpretation of the experiment's purpose and the experimenter's expectations (Orne, 1979). The direction of motion repulsion is opposite to the direction specified by assimilation due to proactive interference (Huang \& Sekuler, 2010), replacement by the subsequent display (Logie, Brockmole, \& Vandenbroucke, 2009), or direction averaging (Treue, Hol, \& Rauber, 2000), three more intuitively obvious expectations an observer might derive about superimposed directions of motion.

Figure 1a illustrates the stimuli and procedure used in Experiment 1 (see also the movie in the online Supplementary Material). We presented two random-dot motion stimuli in sequence whose overall statistical properties were identical, except for the net direction of movement of the dots. Observers had to remember the direction of the first random-dot motion (i.e., the memory display) and then, approximately $2 \mathrm{~s}$ later, had to make a forced choice judgment about the direction of motion of the second random-dot stimulus (i.e., the perception display). Specifically, the observers had to report whether the direction of motion of the perception display was clockwise $(\mathrm{CW})$ or counterclockwise $(\mathrm{CCW})$, relative to a reference bar. Following this perceptual judgment, observers performed a memory recall task by adjusting the angle of a clock hand to the remembered motion direction of the memory display. Immediately after observers' responses on the memory recall task, a green bar provided feedback about the correct motion direction of the memory display. Our results from Experiments 1, 2, and 6 firmly establish that remembered motion can, indeed, bias the appearance of the direction of real visual motion, and a series of additional experiments rule out alternative accounts.

\section{Method}

All observers had normal or corrected-to-normal visual acuity, were 21 to 37 years old, and provided informed consent. Seven observers participated in Experiments 1 and 3, 4 in Experiments 2, 4, and 5, and 6 in Experiment 6. The first author participated in all but Experiment 3, and the second author participated in all the experiments. The other observers were naïve as to the purposes of the experiments and showed effects that were qualitatively similar to those of the authors. a
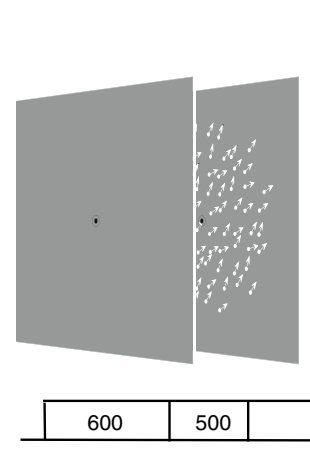

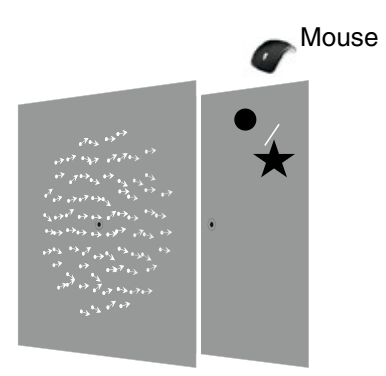

b

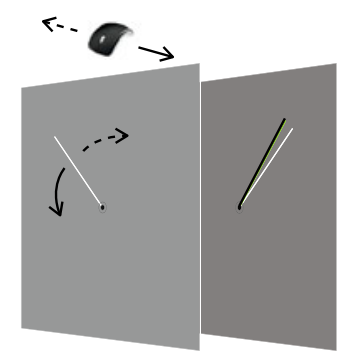

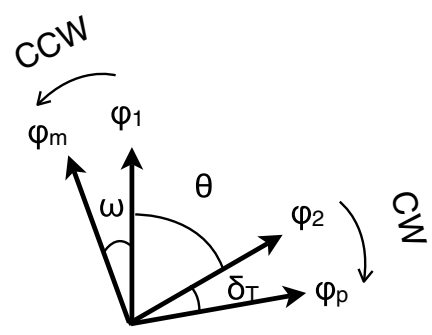

Fig. 1 Illustration of the procedure and measurements in Experiments 1 and 2. a The stimulus sequence consisted of two random-dot motion stimuli and two tasks: the perceptual judgment task and the memory recall task. For the perceptual judgment task, observers indicated whether the direction of moving dots was counterclockwise (CCW) or clockwise $(\mathrm{CW})$ with respect to the reference bar by placing the arrow cursor within a region either $\mathrm{CCW}$ or $\mathrm{CW}$ of the reference bar and then clicking the mouse button. For the memory recall task, observers indicated the direction of the memorized motion by adjusting the angular position of the clock hand. b Illustration of the metrics we used to quantify motion repulsion. The meanings of the symbols are as follows: motion direction of the memory display $\left(\varphi_{1}\right)$, motion direction of the perception display $\left(\varphi_{2}\right)$, perceived motion direction $\left(\varphi_{\mathrm{p}}\right)$, memorized motion direction $\left(\varphi_{\mathrm{m}}\right)$, angular separation between the directions of memory and perception displays $(\theta)$, directional shift in memory representation $(\omega)$, and threshold level shift in perceived motion direction $\left(\delta_{\mathrm{T}}\right)$ 
Stimulus presentation on a Sony GDM-FW900 monitor $(100-\mathrm{Hz}$ frame rate) and the collection of behavioral responses were controlled by the Psychophysics Toolbox (Brainard, 1997; Pelli, 1997). Stimuli were presented on a dark background $\left(0.2 \mathrm{~cd} / \mathrm{m}^{2}\right) 90 \mathrm{~cm}$ in front of the observers in a dimly illuminated room. The fixation point was a circle subtending $0.3^{\circ}$ of visual angle $\left(14 \mathrm{~cd} / \mathrm{m}^{2}\right)$ surrounding a dot. The motion stimulus was a field of approximately 157 dots moving at $3.5^{\circ}$ s, displayed within a circular aperture with a $4^{\circ}$ diameter. Each dot was $0.05^{\circ}$, with a low luminance of $14 \mathrm{~cd} / \mathrm{m}^{2}$ to avoid creating afterimages. The lifetime of each dot was $100 \mathrm{~ms}$, meaning that dots were replotted at random locations every $100 \mathrm{~ms}$, thereby reducing the likelihood that the observer could usefully track a single dot. The directions of the moving dots were normally distributed, with the mean at the designated direction of motion and a standard deviation of 20 angular degrees.

For Experiment 1, the stimulus sequence consisted of two motion stimuli and the two tasks shown in Fig. 1a. Each trial, initiated by the observer's buttonpress, began with a $600-\mathrm{ms}$ fixation period, followed by the $500-\mathrm{ms}$ presentation of the memory display. A 2-s interval with only the fixation point preceded the 500-msec presentation of the perception display. Presented immediately after the offset of that stimulus was a reference bar (a thin white line, $0.03^{\circ}$ wide, $14 \mathrm{~cd} / \mathrm{m}^{2}$ ) subtending $2.7^{\circ}-3.7^{\circ}$ of visual angle from fixation. Observers reported whether the direction of motion was $\mathrm{CCW}$ or $\mathrm{CW}$ with respect to this reference bar by placing the arrow cursor (approximately $0.3^{\circ}$ of visual angle) within a region either $\mathrm{CCW}$ or $\mathrm{CW}$ of the reference bar and then clicking the mouse button. This response had to be made within $2 \mathrm{~s}$ after the onset of the reference bar. One second after the perceptual judgment response, the memory recall task began, in which observers moved a clock hand to indicate the memorized direction of motion from the memory display. The clock hand extended from the outer ring of the fixation point to $2^{\circ}$ of visual angle from the center of the monitor. Observers were required to move the mouse to the left or the right to move the clock hand either $\mathrm{CCW}$ or $\mathrm{CW}$ and then clicked the button to report the remembered direction of motion. Accuracy was stressed, and memory recall was not speeded. The initial position of the clock hand was randomly chosen from the $360^{\circ}$ range. Feedback was provided after the memory recall response in the form of another green clock hand (visible for $500 \mathrm{~ms}$; shown in black in Fig. 1a) indicating the actual direction of motion.

In Experiment 1, two independent variables were parametrically manipulated. One was the angular separation $(\theta)$ between the motion directions of the memory and perception displays, defined as $\theta=\varphi_{1}-\varphi_{2}$. For convention, angle increased in the $\mathrm{CCW}$ direction. Angular separation $\theta$ was manipulated at $\pm 30^{\circ}, \pm 60^{\circ}$, and $\pm 90^{\circ}$, and we determined significance using a regression analysis, because motion repulsion is characteristically maximal at $30^{\circ}$ of separation and decreases linearly with increasing separation. The other independent variable was the angular separation $(\delta)$ between the reference $\left(\varphi_{\mathrm{r}}\right)$ and the motion direction of the perception display $\left(\varphi_{2}\right)$, as defined by $\delta=\varphi_{\mathrm{r}}-\varphi_{2}$. The $\delta$ was varied from $-18^{\circ}$ to $18^{\circ}$ with $6^{\circ}$ step sizes. For each of the four sessions with each observer, the number of measurements at each $\delta$ varied for each angular separation $\theta$ such that there was one measurement for $\delta= \pm 18^{\circ}$, two measurements for $\delta=$ $\pm 12^{\circ}$, four measurements for $\delta= \pm 6^{\circ}$, and eight measurements for $\delta=0^{\circ}$. The memory recall task produced the dependent measure $(\omega)$, which is the difference between the recalled motion direction and actual direction of the first motion stimulus $\left(\omega=\varphi_{\mathrm{m}}-\varphi_{1}\right)$.

Experiment 2 was identical to Experiment 1, except that the angular separation $(\theta)$ covered the entire $360^{\circ}$ range of possible motion directions in $30^{\circ}$ steps. Experiment 3 differed from Experiment 1 only in that there was no memory recall task. In Experiment 4, two perception displays were presented for $500 \mathrm{~ms}$ each, and observers performed a direction judgment task immediately after the offset of each display. The angular separation $(\delta)$ between the motion direction and the reference bar of the first motion display was not correlated with that of the second motion display. We also provided auditory feedback for correct responses only for the first perceptual judgment task in Experiment 4. Experiment 5 was identical to Experiment 1 , except that the memory display was replaced by a static clock hand pointing in a direction corresponding to what had previously been a direction of motion in Experiment 1 ( $2^{\circ}$ in length from the fixation to the outer ring for $500 \mathrm{~ms}$ ). Experiment 6 was identical to Experiment 1, except that the reference bar for the perceptual judgment was presented simultaneously with the random-dot motion stimuli and observers rotated a response knob to indicate the motion direction. To compare the findings across experiments, we used regressions with the between-subjects factor of experiment (two levels; e.g., Experiment 1 vs. 6) and with the within-subjects factor of angular separation $\theta$.

\section{Results}

Memory contaminates perception

Consider a trial on which the perception display moved $\mathrm{CW}$ with respect to the memory display (Fig. 1b). If the motion represented in visual working memory contaminated the appearance of the subsequent perception display, the perceived motion direction in the perception display 
should be shifted CW from the actual direction, and the memorized motion direction should be shifted CCW. To describe the results more efficiently, we collapsed the results across $\mathrm{CW}$ and $\mathrm{CCW}$ relative positions, using the metrics shown in Fig. $1 \mathrm{~b}$.

The results in Fig. 2a revealed perceptual contamination by the memory representation in the form of motion repulsion. Observers perceived a CW shift in the direction of motion relative to the physical motion direction as indicated by leftward shifts in the psychometric functions. The threshold level of the perceived shift $\left(\delta_{\mathrm{T}}\right)$ was obtained from each psychometric curve, using a bootstrapping technique (Wichmann \& Hill, 2001), and those threshold levels are summarized by the circle symbols with solid lines in Fig. 2b. Perceived direction was shifted significantly in the $\mathrm{CW}$ direction for all angular separations $\theta$, as indicated by significance marker $\left({ }^{*}\right)$ obtained from preplanned $t$-tests. The size of the repulsion effect
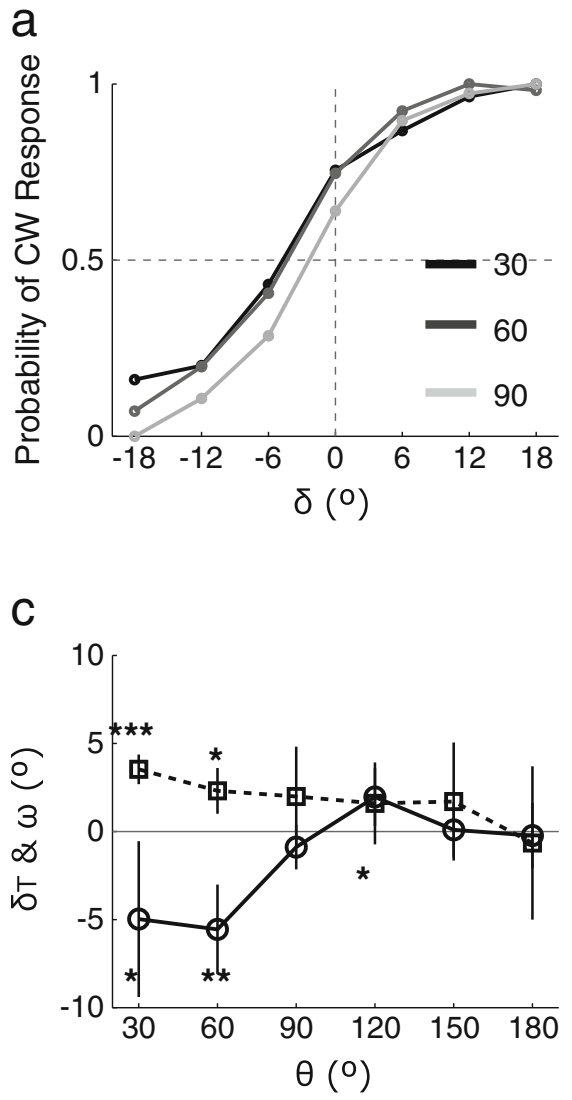

Fig. 2 Results of Experiments 1 and 2. a Averaged psychometric curves of Experiment 1 showing the probability of clockwise (CW) judgment as a function of the angular separation between the reference bar and the motion direction of the perception display. The different gray lines indicate the three angular separations (black $=30^{\circ}$, dark gray $=60^{\circ}$, and light gray $=90^{\circ}$ ). $\mathbf{b}$ The threshold shift in perceived motion direction (circle markers with solid lines) and shift in memory representation (square markers with dotted lines) are plotted as a function of angular separation $\theta$. For perceived motion direction, a was considerably smaller at $90^{\circ}$ of angular separation, as compared with $30^{\circ}$ and $60^{\circ}$ angular separations, as expected due to motion repulsion, $F(1,6)=15.53, \eta^{2}=$ $.72, p<.01$ (results of a simple regression of deviation on angular separation $\theta$ ). This is important because in addition to significant repulsion away from the objective motion signal (i.e., vertical shifts in Fig. 2b), significantly greater deviations in the perceived direction at smaller angular separations is a hallmark of motion repulsion (Marshak \& Sekuler, 1979). The shift in the visual working memory representation $(\omega)$ was obtained by averaging the difference between the recalled motion direction and the motion direction actually shown in the memory display (the square symbols and dotted lines in Fig. 2b). The shifts at the three angular separations, however, did not result in a significant effect of angular separation $\theta, F(1,6)=1.04, \eta^{2}=.15, p=.35$, meaning that the memory representation did not show conspicuous

b

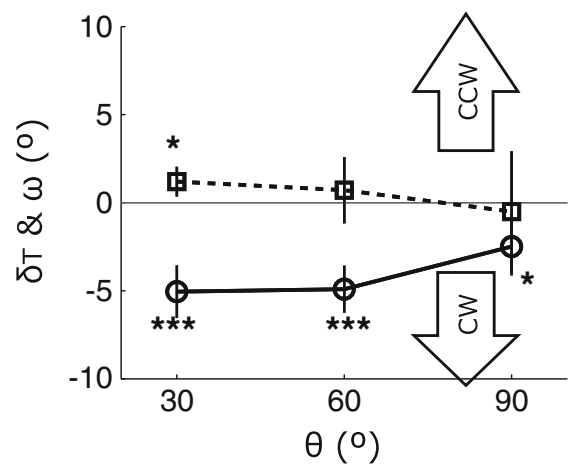

d

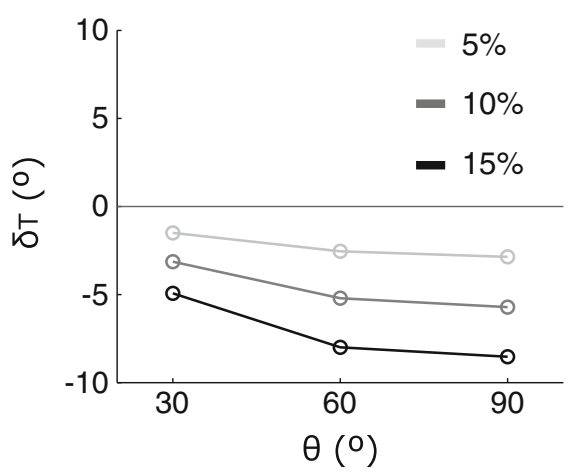

CW shift indicates that repulsion was observed, and for the visual working memory representation, a counterclockwise (CCW) shift indicates that repulsion was observed. Statistical significance obtained from preplanned $t$-test with respect to 0 is indicated by significance symbols $(* p<.05, * * p<.01$, and $* * * p<.001)$. The error bars show the $\pm 95 \%$ confidence intervals. $\mathbf{c}$ Results of Experiment 2 . The figure is plotted using the same conventions as in panel b. d Results of motion aftereffect simulations 
motion repulsion. These results suggest that the shift in the appearance of motion results from the working memory representation pushing the perceptual representation away from the true direction.

\section{Range effects}

We considered whether the repeated measurements within the limited range of angular separations $\theta$ used in Experiment 1 might have allowed observers to predict the direction of motion of the perception display and, thereby, induced a response bias that mimicked motion repulsion. We tested this in Experiment 2 by repeating the procedures of Experiment 1 with angular separations spanning the entire $360^{\circ}$ range of motion directions. Figure $2 \mathrm{c}$ shows that motion repulsion for the perception display was robustly reproduced at small angular separations $\left(30^{\circ}\right.$ and $\left.60^{\circ}\right)$ and the magnitude of repulsion varied significantly across angular separations, as shown by a significant effect of $\theta$ on the accuracy of the perceptual judgment, $F(1,3)=$ $34.46, \eta^{2}=.92, p<.01$. In addition, the regression on memory recall performance also showed a significant repulsion effect, $F(1,3)=68.85, \eta^{2}=.96, p<.01$, induced by the perception display on the representation of the memory display. Evidently, the absence of a robust repulsion effect in the memory recall task in Experiment 1 occurred because the motion direction of the perception display provided predictive information that allowed observers to infer the memorized direction, due to the limited angular separation range. Note also that the repulsion effect we observed in Experiment 2, approximately $10^{\circ}$, is comparable to the size of perceptual repulsion reported previously (Rauber \& Treue, 1999), demonstrating the robustness of the repulsion effect exerted by the working memory representation.

\section{Visual adaptation}

Might our findings be due to motion adaptation? Note that an aftereffect induced by motion adaptation after the memory display has been viewed could produce a repulsion-like effect. However, the results from Experiments 1 and 2 cannot be explained by motion adaptation, for the following two reasons. First, the 500-msec presentation of the random-dot motion we used was too brief to induce a strong motion aftereffect (Blake \& Hiris, 1993). Moreover, adaptation could not build up, because the direction of motion in the memory display varied randomly over trials. Second, if our effects had been due to a motion aftereffect, we would have obtained the opposite pattern of results. Specifically, motion adaptation predicts that the size of the repulsion effect at the $30^{\circ}$ angular separation should be smaller than the effect size at $60^{\circ}$ and $90^{\circ}$. Figure $2 \mathrm{~d}$ shows the results of modeling in which repulsion magnitude was predicted by a simple summation of the motion vector of the perception display and a motion vector associated with the motion aftereffect of the memory display $(5 \%, 10 \%$, and $15 \%$ of aftereffect magnitude). Our results in Experiments 1 and 2 are clearly opposite to those that would have been produced by the motion aftereffect.

Eye movements

Previous studies have shown that smooth pursuit eye movements can impair performance on a short-term memory span task involving judgments of spatial location (e.g., D. Pearson \& Sahraie, 2003; Postle, Idzikowski, Sala, Logie, \& Baddeley, 2006). Is it possible that the random-dot motion in our 500-ms displays evoked pursuit eye movements and, therefore, diluted the effect of working memory on subsequent visual perception of motion direction? Even if there were unintended eye movements despite the instruction of strict fixation, we are disinclined to believe that they could have produced the pattern of results we found. First, Postle et al. (2006) showed that it is voluntary eye movement control, not the movements per se, that causes the deleterious effects of eye movements on visuospatial working memory. Second, Postle et al. also found that effects of voluntary eye movements were seen only on tasks that involved remembering spatial locations, a very different kind of judgment from the motion direction task. Third and finally, several studies of perceptual motion repulsion have found that eye movements play essentially no role in mediating motion repulsion (Marshak \& Sekuler, 1979; Rauber \& Treue, 1999).

\section{Visual priming}

One could argue that visual priming produced by the mere presentation of the memory display, rather than its active maintenance in visual working memory, might produce the observed repulsion effect. It should be noted that the priming literature would imply that observers will experience assimilation instead of repulsion (Pinkus \& Pantle, 1997). However, to test this priming account empirically, we conducted Experiment 3, in which we presented two motion stimuli in exactly the same way as we did in Experiment 1, but without a memory recall task for the memory display of Experiment 1 (Fig. 3a). Threshold levels of the perceived motion direction $\left(\delta_{\mathrm{T}}\right)$ across all angular separations were not different from 0 on the basis of preplanned $t$-tests, and they were equivalent for all three angular separations, as indicated by a nonsignificant regression on the effect of angular separation $\theta, F(1,6)=1.77, \eta^{2}=.23, p=.23$ (Fig. 3b). However, 
a

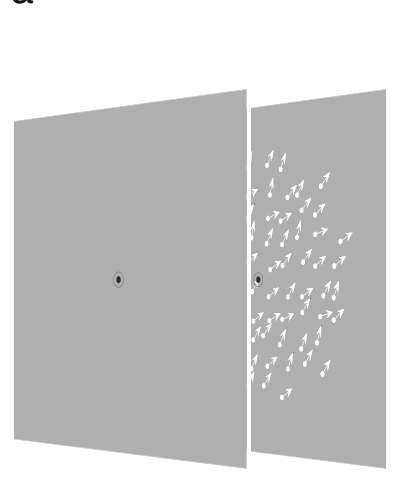

600

\begin{tabular}{|l|l|l|l|l|}
\hline 600 & 500 & 2000 & 500 & less than 2000 \\
\hline
\end{tabular}

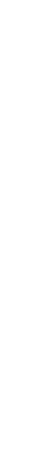

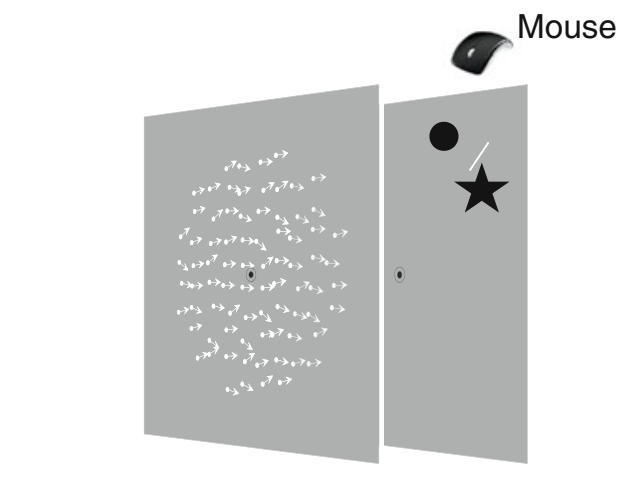

Time (msec)

Fig. 3 Procedure and results of Experiment 3. a Illustration of an example trial in Experiment 3, in which we presented two motion stimuli in exactly the same way as in Experiment 1, but without a

perceived motion directions obtained in Experiment 1 were significantly different from those in this experiment, as evidenced by a significant main effect of experiment on perceived direction, $F(1,6)=30.03, \eta^{2}=.83, p<.01$. Thus, visual priming cannot explain the systematic shift in perceived motion direction observed in Experiment 1.

Two tasks, no memory demands

Another rival account of the motion repulsion effect is that it resulted from the general task demands associated with the initial display being task relevant, whether or not it had to be stored in memory. To evaluate that possibility in Experiment 4, we required observers to perform two successive perceptual judgments, instead of probing memory and perception (Fig. 4a). Thus, the stimulus arrays in Experiment 4 were identical to those in Experiment 1, but the tasks differed. Observers reported whether the direction of motion was $\mathrm{CW}$ or $\mathrm{CCW}$ relative to the reference bar for each stimulus in exactly the same way as they did for just the second motion display in Experiment 1. Angular separation $(\theta)$ was defined between the two motion directions of the successive perception displays. As is shown in Fig. 4b, threshold levels of the perceived motion direction $\left(\delta_{\mathrm{T}}\right)$ of the second perception display were not different from 0 for all angular separations $(\theta)$, and they were equivalent for all three angular separations, resulting in a nonsignificant regression, $F(1,3)=0.4714, \eta^{2}=.09, p>.5$. Nevertheless, perceived motion directions in Experiment 1 were significantly different from those in Experiment $4, F(1,3)=$ 26.64, $\eta^{2}=.90, p<.05$, meaning that the general task demands of the first stimulus being task relevant cannot b

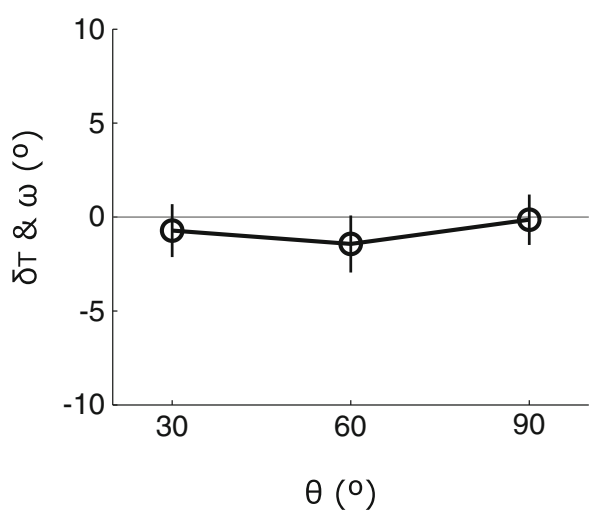

memory recall task. b Results of Experiment 3 using the same conventions as in Fig. 2b, showing the results of just the perceptual judgment (circle symbols) after simple priming explain deviation of the perceived motion direction in Experiment 1.

\section{Remembering a vector}

One might argue that the memory task is accomplished by retaining an abstract vector representation of the motion display, with that vector instigating the subsequently measured motion repulsion and not remembered motion per se. If motion repulsion is being produced by a vector representation in memory, motion repulsion caused by two simultaneously visible random-dot motion displays would likely be due to very different neural mechanisms than the repulsion we observed here (Marshak \& Sekuler, 1979).

To determine whether a mnemonic vector representation of perceived motion direction can indeed produce repulsion, we performed Experiment 5, which was identical to Experiment 1, with one important exception. We replaced the memory display with a clock hand and required observers to remember the orientation of that clock hand (Fig. 5a). The angular separation $(\theta)$ was defined by the difference between the orientation of this clock hand and the motion direction of the perception display. Neither perceived motion direction nor recalled clock hand direction (Fig. 5b) was significantly different from 0 at any angular separation, and the magnitude of repulsion was not statistically different among the different angular separations $F(1,3)=3.17, \eta^{2}=.51, p=.17$, for perceived motion, and $F(1,3)=0.06, \eta^{2}=.02, p>.5$, for memorized clock hand direction]. Yet, the perceived motion directions differed significantly between Experiments 1 and $5, F(1,3)=18.56$, $\eta^{2}=.86, p<.05$. Thus, we are confident that the repulsion 
a

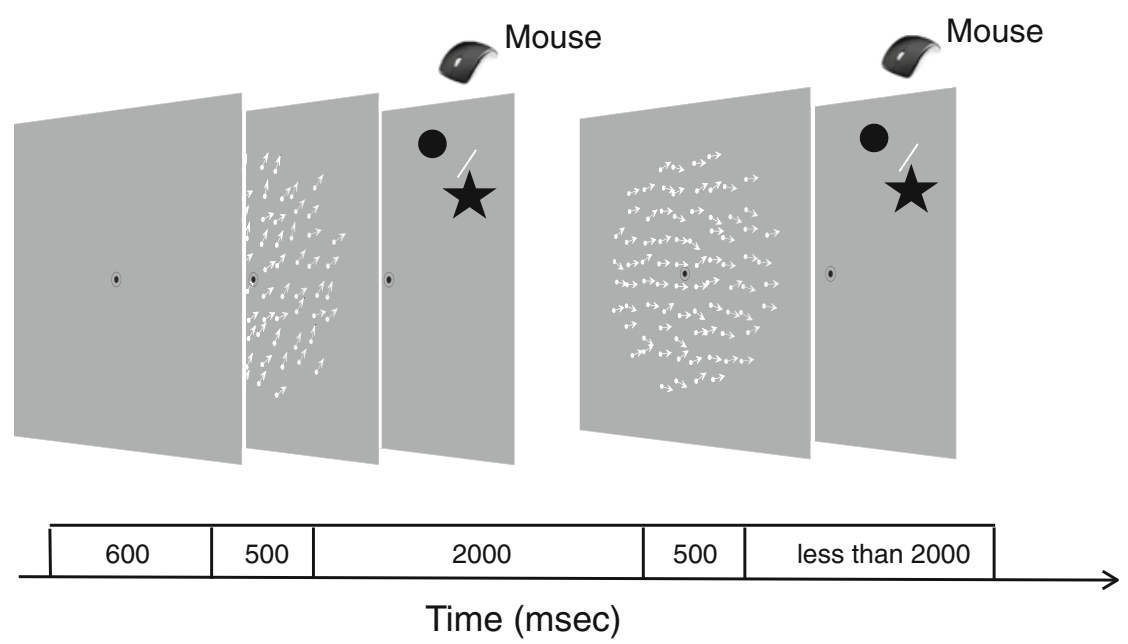

b

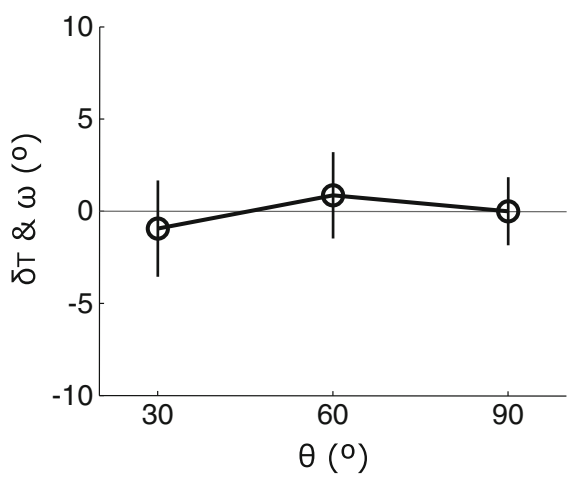

Fig. 4 Procedure and results of Experiment 4. a Illustration of an example trial from Experiment 4, in which observers performed two successive perceptual judgment tasks. b Results of Experiment 4, showing the precision of the second perceptual judgment (as in Fig. 2b)

effects we have observed cannot be chalked up to the influence of a simple mnemonic representation of direction, like a vector, altering the perceived direction of motion of the subsequently viewed test display.

Eliminating the memory demands of the perceptual judgment

Finally, one might argue that the perceptual task in our previous experiments actually relied on visual working memory. According to this argument, the repulsion effect we measured was between two working memory representations (i.e., of the memory and perception displays), because observers were making their judgments and responses to the perceived motion direction after the stimulus was no longer visible. To test this possibility, we presented the reference bar simultaneously with the perception display and required observers to perform the $\mathrm{CW}$ versus $\mathrm{CCW}$ judgment with a response knob (e.g., rotate the knob $\mathrm{CW}$ when motion direction is $\mathrm{CW}$ with respect to the reference bar) so that they could respond to the actual visual appearance of the perception display while it was still visible, thereby removing the memory demands in the perceptual task (Fig. 6a). All other aspects of Experiment 6 were identical to those in Experiment 1 . The results of Experiment 6 replicated the perceptual motion repulsion effect even though this speeded task was relatively difficult according to our observers. We found perceived shifts at $30^{\circ}$ and $60^{\circ}$ angular separations that were significantly different from 0 , and their magnitudes a
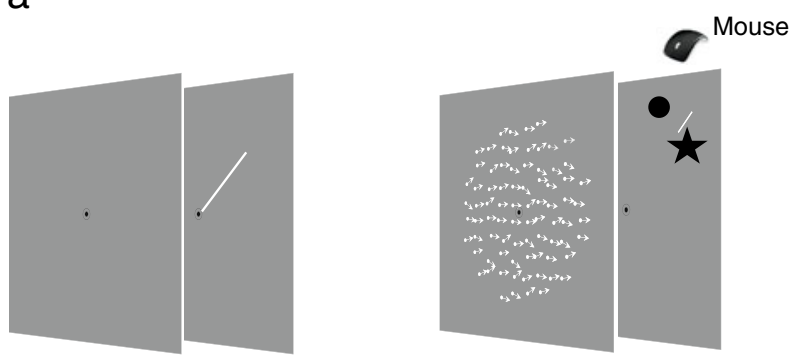

b

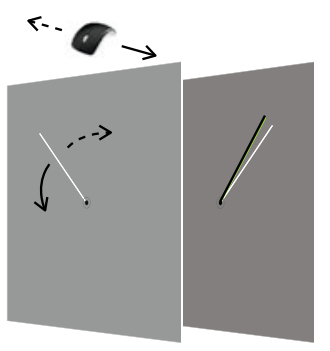

\begin{tabular}{|l|l|}
\hline until response & 500 \\
\hline
\end{tabular}

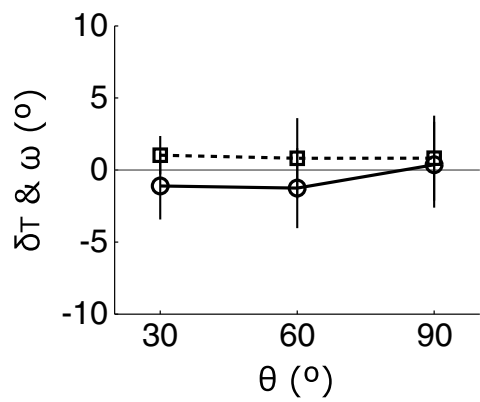

Time (msec)

Fig. 5 Procedure and results of Experiment 5. a Illustration of an example trial of Experiment 5 in which the memory display in Experiment 1 was replaced by clock hand of a certain orientation. Observers were asked to recall the orientation of this clock hand, in addition to performing the motion judgment task on the perception display. b Results of Experiment 5 using the same conventions as in Fig. $2 b$ 
a

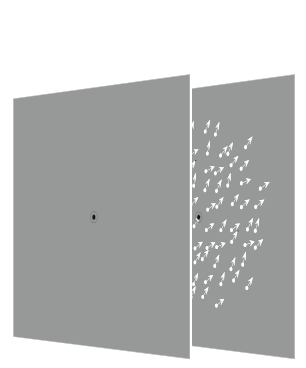

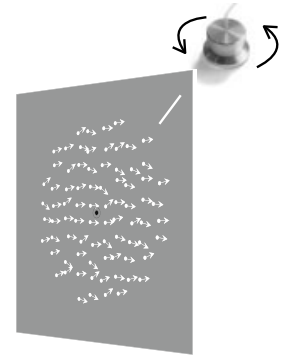

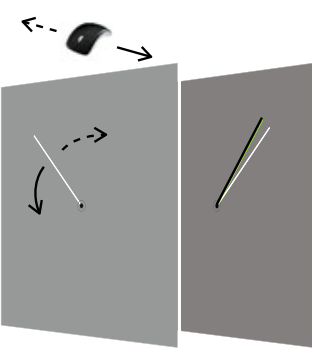

\begin{tabular}{l|l|l|l|l|l|l|l|}
\hline 600 & 500 & 2000 & 500 & less than 2000 & 1000 & until response & 500 \\
\hline
\end{tabular}

b

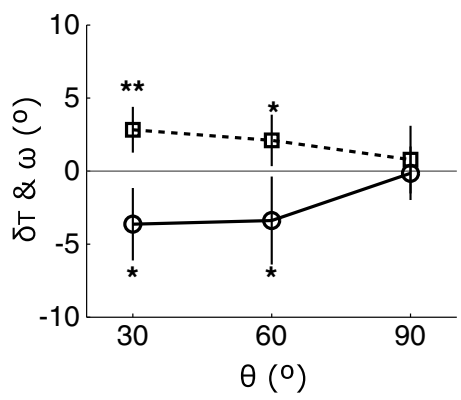

Fig. 6 Procedure and results of Experiment 6. a Illustration of an example trial in Experiment 6, which was identical to Experiment 1 except that the reference bar was simultaneously presented with the perception display. Observers were required to perform the clockwise/ counterclockwise judgment with a response knob (e.g., rotate the knob $\mathrm{CW}$ when motion direction was $\mathrm{CW}$ with respect to the reference bar). b Results of Experiment 6 using the same conventions as in Fig. 2b

observed between a visual working memory representation and a new moving stimulus implies that genuine visual features represented in working memory can interact with and influence the appearance of newly presented sensory information. ${ }^{1}$

How is this interaction mediated? Earlier work pointed to a strong influence of visual working memory on attentional selection (Olivers, Peters, Houtkamp, \& Roelfsema, 2011). Here, we show that visual working memory's influence can penetrate deep enough into the stream of events underlying perception that the contents of memory can literally change the appearance of what we are viewing. It seems logical to conclude that this influence arises because working memory co-opts the neural machinery involved in sensory processing. Indeed, we know from recent neurophysiology and neuroimaging studies that visual working memory representations may be maintained for several seconds in early visual areas (e.g., V1, $\mathrm{V} 2, \mathrm{~V} 3$, and V4) where the analysis of basic visual information occurs (Harrison \& Tong, 2009; Serences et al., 2009; Supèr, Spekreijse, \& Lamme, 2001). There is an abundance of evidence indicating that imagining a visual stimulus can influence subsequent visual perception of related visual stimuli (Farah, 1985; Ishai \& Sagi, 1995; Kosslyn, Ganis, \& Thompson 2001; Pearson, Clifford, \& Tong, 2008; Perky, 1910). One could be led to the belief that the neural concomitants of visual imagery may be found within visual working memory, meaning that our effects are similar in their source. However, this view disregards recent evidence that visual perception influences imagery but not visual working memory (e.g.,

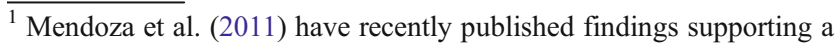
conclusion similar to ours.
} 
Andrade, Kemps, Werniers, May, \& Szmalec, 2002; Baddeley, 2007), suggesting that imagery and visual working memory have different substrates that interact with perception in different ways.

Finally, our results may have important practical implications. There are numerous instances where we engage in visually guided activities (e.g., driving) while rehashing visual events in our mind's eye. Common sense tells us that this mental replay is harmless in that it does not interfere with our ability to register and react to objects within our visual field. Evidently, however, that is not always true when the contents of our working memories overlap with the contents of our perceptual world.

Author Note We thank Robert Sekuler and two anonymous reviewers for valuable comments. This research was supported by grants from the National Institutes of Health (RO1-EY019882; P30EY008126), from the National Science Foundation (BCS 09-57072), and from the World Class University Initiative funded by the National Research Foundation of Korea and the Ministry of Education, Science and Technology (R32-10142).

\section{References}

Andrade, J., Kemps, E., Werniers, Y., May, J., \& Szmalec, A. (2002). Insensitivity of visual short-term memory to irrelevant visual information. Quarterly Journal of Experimental Psychology, 55A, 753-774. doi:10.1080/02724980143000541

Baddeley, A. D. (2007). Working memory, thought, and action. New York: Oxford University Press.

Blake, R., Cepeda, N. J., \& Hiris, E. (1997). Memory for visual motion. Journal of Experimental Psychology. Human Perception and Performance, 23, 353-369.

Blake, R., \& Hiris, E. (1993). Another means for measuring the motion aftereffect. Vision Research, 33, 1589-1592.

Brainard, D. H. (1997). The Psychophysics Toolbox. Spatial Vision, $10,433-436$

Desimone, R., \& Duncan, J. (1995). Neural mechanisms of selective visual attention. Annual Review of Neuroscience, 18, 193-222. doi:10.1146/annurev.ne.18.030195.001205

Farah, M. J. (1985). Psychophysical evidence for a shared representational medium for mental images and percepts. Journal of Experimental Psychology. General, 114, 91-103.

Fukuda, K., Vogel, E., Mayr, U., \& Awh, E. (2010). Quantity, not quality: The relationship between fluid intelligence and working memory capacity. Psychonomic Bulletin Review, 17, 673-679. doi:10.3758/17.5.673

Harrison, S. A., \& Tong, F. (2009). Decoding reveals the contents of visual working memory in early visual areas. Nature, 458, 632635. doi:10.1038/nature 07832

Hiris, E., \& Blake, R. (1996). Direction repulsion in motion transparency. Visual Neuroscience, 13, 187-197.

Huang, J., \& Sekuler, R. (2010). Distortions in recall from visual memory: Two classes of attractors at work. Journal of Vision, 10 (2, Art. 24), 1-27. doi:10.1167/10.2.24

Ishai, A., \& Sagi, D. (1995). Common mechanisms of visual imagery and perception. Science, 268, 1772-1774.

Kosslyn, S. M., Ganis, G., \& Thompson, W. L. (2001). Neural foundations of imagery. Nature Neuroscience, 2, 635-642.
Logie, R. H. (1986). Visuo-spatial processing in working memory. Quarterly Journal of Experimental Psychology, 38A, 229247.

Logie, R. H., Brockmole, J. R., \& Vandenbroucke, A. R. E. (2009). Bound feature combinations in visual short-term memory are fragile but influence long-term learning. Visual Cognition, 17, 160-179.

Luck, S. J., \& Vogel, E. K. (1997). The capacity of visual working memory for features and conjunctions. Nature, 390, 279-281. doi: $10.1038 / 36846$

Marshak, W., \& Sekuler, R. (1979). Mutual repulsion between moving visual targets. Science, 205, 1399-1401.

Mendoza, D., Schneiderman, M., Kaul, C., \& Martinez-Trujillo, J. (2011). Combined effects of feature-based working memory and feature-based attention on the perception of visual motion direction. Journal of Vision, 11(1):11, 1-15.

Miller, G. A. (1956). The magical number seven plus or minus two: Some limits on our capacity for processing information. Psychological Review, 63, 81-97.

Olivers, C. N. L., Peters, J., Houtkamp, R., \& Roelfsema, P. R. (2011). Different states in visual working memory: When it guides attention and when it does not. Trends in Cognitive Sciences.

Orne, M. T. (1979). Social psychology of the psychological experiment: With particular reference to demand characteristics and their implications. American Psychologist, 17, 776-783.

Panzeri, S., Brunel, N., Logothetis, N. K., \& Kayser, C. (2010). Sensory neural codes using multiplexed temporal scales. Trends in Cognitive Sciences, 33, 111-120. doi:10.1016/j.tins. 2009.12.001

Pasternak, T., \& Greenlee, M. W. (2005). Working memory in primate sensory systems. Nature Reviews Neuroscience, 6, 97-107. doi:10.1038/nrn1603

Pearson, D., \& Sahraie, A. (2003). Oculomotor control and the maintenance of spatially and temporally distributed events in visuo-spatial working memory. Quarterly Journal of Experimental Psychology, 56A, 1089-1111.

Pearson, J., Clifford, C. W. G., \& Tong, F. (2008). The functional impact of mental imagery on conscious perception. Current Biology, 18, 982-986. doi:10.1016/j.cub.2008.05.048

Pelli, D. G. (1997). The VideoToolbox software for visual psychophysics: Transforming numbers into movies. Spatial Vision, 10, 437-442.

Perky, C. W. (1910). An experimental study of imagination. The American Journal of Psychology, 21, 422-452.

Pinkus, A., \& Pantle, A. (1997). Probing visual motion signals with a priming paradigm. Vision Research, 37, 541-552.

Postle, B. R., Idzikowski, C., Sala, S. D., Logie, R. H., \& Baddeley, A. D. (2006). The selective disruption of spatial working memory by eye movements. Quarterly Journal of Experimental Psychology, 59, $100-120$.

Quinn, J. G., \& McConnell, J. (1999). Manipulation of interference in the passive visual store. European Journal of Cognitive Psychology, $11,373-389$

Rauber, H. J., \& Treue, S. (1999). Revisiting motion repulsion: Evidence for a general phenomenon? Vision Research, 39, 31873196.

Schmeichel, B. J., \& Demaree, H. A. (2010). Working memory capacity and spontaneous emotion regulation: High capacity predicts self-enhancement in response to negative feedback. Emotion, 10, 739-744. doi:10.1037/a0019355

Serences, J. T., Ester, E. F., Vogel, E. K., \& Awh, E. (2009). Stimulusspecific delay activity in human primary visual cortex. Psychological Science, 20, 207-214. doi:10.1111/j.14679280.2009.02276.x

Soto, D., Hodsoll, J., Rotshtein, P., \& Humphreys, G. W. (2008). Automatic guidance of attention from working memory. Trends 
in Cognitive Sciences, 12, 342-348. doi:10.1016/j.tics. 2008.05.007

Supèr, H., Spekreijse, H., \& Lamme, V. A. (2001). A neural correlate of working memory in the monkey primary visual cortex. Science, 293, 120-124. doi:10.1126/science. 1060496

Treue, S., Hol, K., \& Rauber, H. J. (2000). Seeing multiple directions of motion-physiology and psychophysics. Nature Neuroscience, 3, 270-276. doi:10.1038/72985
Wichmann, F. A., \& Hill, N. J. (2001). The psychometric function: I. Fitting, sampling, and goodness of fit. Perception \& Psychophysics, 63, 1293-1313.

Woodman, G. F., \& Luck, S. J. (2007). Do the contents of visual working memory automatically influence attentional selection during visual search? Journal of Experimental Psychology. Human Perception and Performance, 33, 363-377. doi:10.1037/0096-1523.33.2.363 